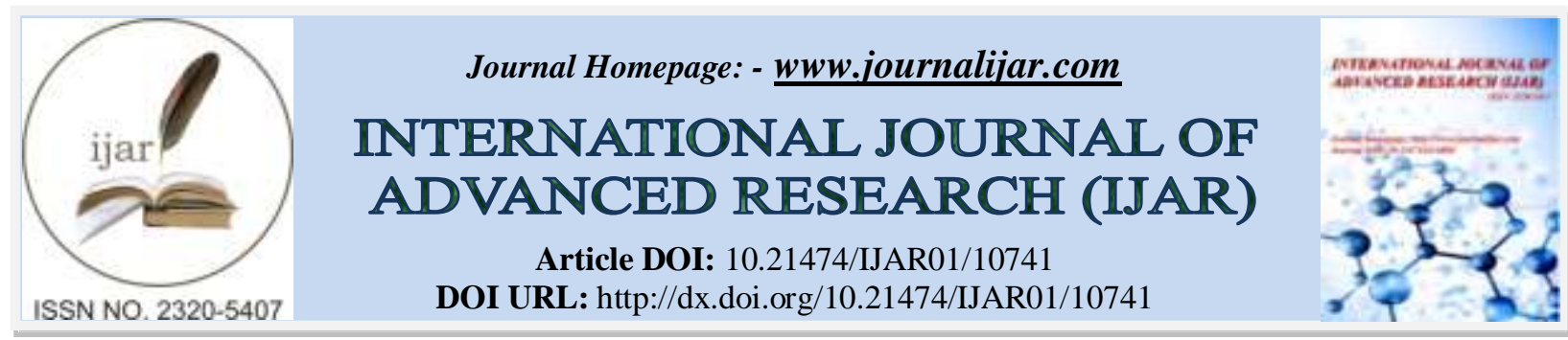

\title{
RESEARCH ARTICLE \\ SUBMUCOSAL DIATHERMY VS. COBLATION PARTIAL TURBINECTOMY ON NASAL MUCOCILIARY FUNCTION
}

Merwin Paul. R, K. Shoba and B. C. Surekha

Department of Otorhinolaryngology, Saveetha Medical College and Hospital, Chennai.

\section{Manuscript Info}

Manuscript History

Received: 05 February 2020

Final Accepted: 07 March 2020

Published: April 2020

\section{Abstract}

Introduction: Mucociliary function is more important in the inferior turbinate than the other turbinates. Hypertrophy of the inferior turbinate is one of the commonest reason for chronic nasal obstruction. Mucociliary function is a vital defence mechanism that protects the respiratory system against microbes and alternative foreign particles. Nasal mucociliary function is either primarily or secondarily impaired within the majority of patients with repeated chronic rhinitis, sinusitis. A number of interventions are offered for the treatment of nasal obstruction secondary to inferior turbinate hypertrophy. An ideal procedure for turbinate reduction is one that ought to be related to least discomfort or adverse reactions and will preserve the physiological function of the turbinate, like control of humidification and temperature of the inspired air. Hence this study was done with the following aim Aim: The aim of this study is to evaluate nasal functions after treatment by submucosal diathermy and coblation partial turbinectomy on chronic nasal airway obstruction due to inferior turbinate hypertrophy.

Methods: This study was started after obtaining the approval of Institutional Ethics Committee. Patients with unilateral or bilateral nasal block who visited the Department of Otorhinolaryngology, SMC\&H, were screened. Of them, 72 patients with inferior turbinate hypertrophy as the cause for the nasal block had been recruited into the study after obtaining their written informed consent. All the patients were registered into the study during their first visit and all the patients were followed-up at 3 months and 6 months. The 72 patients who were recruited based on the inclusion and exclusion criteria were randomized using simple randomization into 2 treatment groups ie. Thirty six patients in group A - submucosal diathermy and thirty six patients in group B - coblation partial turbinectomy. A detailed history, examination and routine investigations were done on all the patients who were enrolled in the study. Nasal mucociliary function by saccharin test and Peak Nasal Inspiratory Flow Rate (PNIF) have been measured in all the patients before the surgery and at 3 months and 6 months post operatively.

Results \& Conclusion: Both the surgical techniques showed a statistically significant improvement in nasal mucociliary function and airway patency three months and 6 months post operatively. There was 
no statistically significant difference between the two surgical procedures. This suggests that both submucosal diathermy and coblation partial turbinectomy are equally effective in improving nasal mucociliary function and airway patency due to inferior turbinate hypertrophy.

Copy Right, IJAR, 2020,. All rights reserved.

\section{Introduction:-}

Inferior turbinate is the workhouse of the nasal cavity. It is bulkier than the other turbinates and it is not a part of ethmoid bone. Mucociliary function is more important in the inferior turbinate than the other turbinates. Hypertrophy of the inferior turbinate is one of the commonest reason for chronic nasal obstruction. The physiological "nasal valve," initial termed by Mink in 1920, has been studied extensively and located to exist close to the junction of the cartilaginous vestibule and bony nasal pyramid. At this site, the anterior facet of the enlarged inferior turbinate exerts its impact on nasal air flow. ${ }^{1}$ A number of the other common causes of inferior turbinate hypertrophy including compensative enlargement ensuing from a long-standing septal deviation and also as generalized nasal mucosal diseases in allergic and vasomotor rhinitis. ${ }^{2}$

Mucociliary function is a vital defence mechanism that protects the respiratory system against microbes and alternative foreign particles. Nasal mucociliary function is either primarily or secondarily impaired within the majority of patients with repeated chronic rhinitis, sinusitis. ${ }^{3}$ Measurements of nasal mucociliary function are ordinarily performed as a part of the analysis of patients with symptoms of the upper and lower respiratory tract more so in the case of nasal mucociliary function is impaired in the majority of surgical reductions of the inferior turbinate. ${ }^{3}$

Rhinomanometry (RM) is considered as the benchmark for measurement of nasal airway resistances. Though RM is the customary technique for the assessment of nasal resistance, peak nasal inspiratory flow (PNIF) has been shown to be extremely useful with nasal airway resistances. It is reproducible in the analysis of nasal airway obstruction, an objective nasal patency as rhinomanometry. ${ }^{4}$ Moreover, PNIF is a low-cost, simple, easily performed technique to assess nasal patency, and it is applicable for serial measurements.

A number of interventions are offered for the treatment of nasal obstruction secondary to inferior turbinate hypertrophy. They include antihistamines, decongestants, corticosteroid turbinate injections, cryosurgery, electrocautery, total or partial turbinectomy, turbinoplasty, submucous resection, submucosal diathermy, laserassisted turbinoplasty, coblationturbinectomy and radiofrequency energy tissue ablation. ${ }^{1,5,6}$

The goal of the surgical procedure ought to diminish complaints with optimum volume reduction while preservation of nasal function. ${ }^{7}$

An ideal procedure for turbinate reduction is one that ought to be related to least discomfort or adverse reactions and will preserve the physiological function of the turbinate, like control of humidification and temperature of the inspired air. $^{2}$

The variety of surgical techniques widely practiced indicates the dearth of agreement on the optimal technique. Hence this study was done with following objectives.

\section{Aim and Objectives:-}

Aim:

The aim of this study is to evaluate nasal functions after treatment by submucosal diathermy and coblation partial turbinectomy on chronic nasal airway obstruction due to inferior turbinate hypertrophy.

\section{Objectives:-}

To evaluate the nasal mucociliary function after treatment by submucosal diathermy and coblation partial turbinectomy on chronic nasal airway obstruction due to inferior turbinate hypertrophy. 
To evaluate the airway patency after treatment by submucosal diathermy and coblation partial turbinectomy on chronic nasal airway obstruction due to inferior turbinate hypertrophy.

\section{Materials and Methods:- Design and Setting:}

This is a prospective randomized controlled study and was undertaken in the Department of Otorhinolaryngology, Saveetha Medical College from March 2018 to September 2019. The study compares the effectiveness of submucosal diathermy and coblation partial turbinectomy in improving nasal mucociliary function in patients with chronic nasal obstruction due to inferior turbinate hypertrophy.

\section{Participants:}

All patients suspected or confirmed to have chronic nasal airway obstruction due to inferior turbinate hypertrophy, as evidenced by nasal mucociliary function and airway patency tests were included in this prospective randomized controlled trial, after obtaining written informed consent. Relevant clinical history and examination were done for all the study participants.

\section{Inclusion criteria:}

1. Adult patients ( $>18$ years) of both gender

2. Patients having unilateral or bilateral inferior turbinate hypertrophy with chronic nasal obstruction

\section{Exclusion criteria:}

1. History of previous nasal surgeries

2. Prolonged bleeding time and clotting time

3. Active nasal infection

4. Patients diagnosed with Kartagener's syndrome/ Young's syndrome and other mucociliary dysfunction.

\section{Methodology:-}

The study was started after obtaining the approval of Institutional Ethics Committee. Patients with unilateral or bilateral nasal block who visited the Department of Otorhinolaryngology, SMC\&H, were screened. Of them, 72 patients with inferior turbinate hypertrophy as the cause for the nasal block had been recruited into the study after obtaining their written informed consent. All the patients were registered into the study during their first visit and all the patients were followed-up at 3 months and 6 months.

Clinically Cottle's test, Cotton wool test and Cold spatula test were done to rule out other causes of nasal block. Radiological examination of CT paranasal sinuses were done and the patients were advised about the need for surgery and were explained about the surgical procedure.

The 72 patients who were recruited based on the inclusion and exclusion criteria were randomized using simple randomization into 2 treatment groups ie. Thirty six patients in group A - submucosal diathermy and thirty six patients in group B - coblation partial turbinectomy

All the patients enrolled in a particular study group were explained about the pros and cons of the surgery. A detailed history, examination and routine investigations like complete blood count, random blood sugar, renal function test, liver function test, urine routine, electrolytes, HIV, Hbs Ag, HCV, blood grouping \& typing, bleeding time, clotting time, chest x-ray, ECG, PT, aPTT, INR were done on all the patients who were enrolled in the study. Anesthetic fitness was sought for all the patients prior to surgery.

Nasal mucociliary function by saccharin test and Peak Nasal Inspiratory Flow Rate (PNIF) have been measured in all the patients before the surgery and at 3 months and 6 months post operatively.

\section{Saccharin test:}

This test was carried out in the out-patient department. Patient was made to sit erect with 10 degree flexion of the neck. A small piece of saccharin of size $1 \mathrm{~mm}$ was placed under direct vision on the inferior turbinate $1 \mathrm{~cm}$ away from the anterior end. The patients were instructed not to spit, sneeze or cough during the test duration. After 
placing the saccharin particle, the time taken for the patient to perceive the sweet taste of saccharin the throat was recorded in minutes.

\section{Peak Nasal Inspiratory Flow:}

A portable Youlten peak flow meter (element Clark international) was used for the measurement of PNIF. The mask attached to the peak flow meter were chosen to fit tightly on each patient's face without touching the nose. The masks were cleaned with alcohol swabs and dried between the uses for the next patient.

All the subjects were tested while sitting and the nostril to be tested is left free and the other nostril was sealed off with an adhesive tape (microfoam $3 \mathrm{~mm}$ ) and tested. The patients were encouraged to inhale as hard and fast as they could through the mask keeping the mouth closed and starting from the end of a full expiration. Three satisfactory normal inspirations were obtained and the greatest of the three results was taken as the PNIF value.

\section{Surgery:}

All the patients were prepared before the day of surgery by giving intravenous antibiotics, Inj. TT $0.5 \mathrm{ml}$ intramuscular stat, Inj. Lignocaine $0.1 \mathrm{ml}$ intradermal as test dose, Inj. Pantoprazole $40 \mathrm{mg}$ iv, Tab. Alprazolam 0.2 mg P/O HS, Tab. Metoclopramide $10 \mathrm{mg}$ P/O HS. In case of male patients, their moustache was shaved. Written informed consent was taken from the patient and their attender for the surgery. All the surgeries were performed by a single surgeon.

\section{Coblation Partial Turbinectomy:}

Under general anesthesia, the patients were placed in supine position, parts were painted and draped. Turbinate hypertrophy was visualised and infiltration with normal saline was done at the anterior end of the inferior turbinate. The self-irrigating turbinator (coblator wand) was introduced into the submucous layer of the inferior turbinate through its anterior end to the desired length without touching the bone. The turbinator was withdrawn slowly, coblating the submucous layer, which leads to reduction in the size of the turbinate. This procedure was repeated until satisfactory reduction in the turbinate size was achieved. Care was taken so as to prevent damage to the mucosa and the bone. Nasal packing and dressing were done and the patient was extubated after achieving complete hemostasis. Post operatively, antibiotics and analgesics were given to all the patients.

\section{Submucosal Diathermy:}

Similarly a diathermy was introduced into the submucosal layer of the inferior turbinate through the anterior end and cauterised by slowly withdrawing the diathermy. This procedure was repeated until satisfactory reduction in the turbinate size was achieved.

\section{Statistical Analysis:}

The data collected by the above methodology were analysed statistically using SPSS version 20. The nasal mucociliary function and PNIF values were compared using Repeated Measures of ANOVA within the group and using Mann Whitney test between the two groups. A p value of $<0.05$ was considered statistically significant.

\section{Results:-}

Demographic data and description:

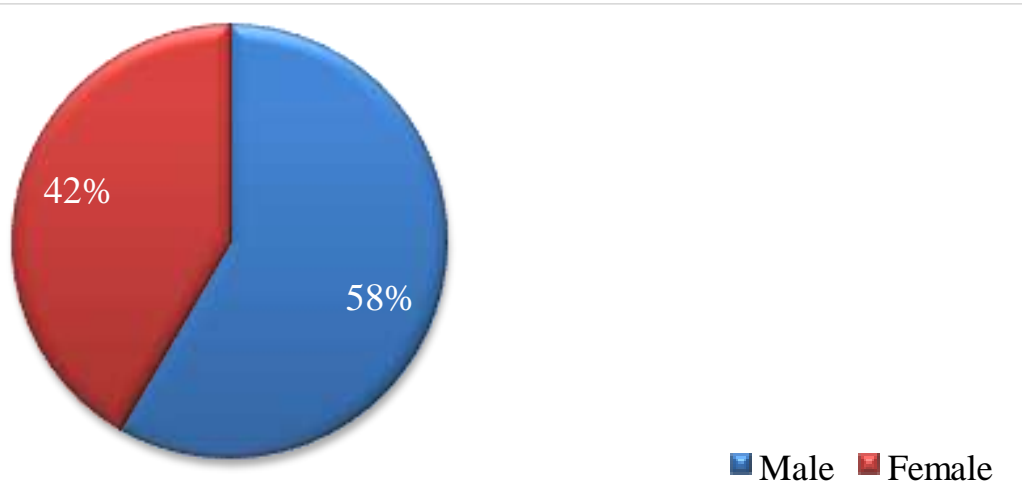

Fig 1:- Gender distribution in the study population. 
Fig. 1 shows the percentage of gender distribution in the study patients. In our study of the seventy two patients, $58 \%(\mathrm{n}=42)$ were male and $42 \%(\mathrm{n}=30)$ were female patients.

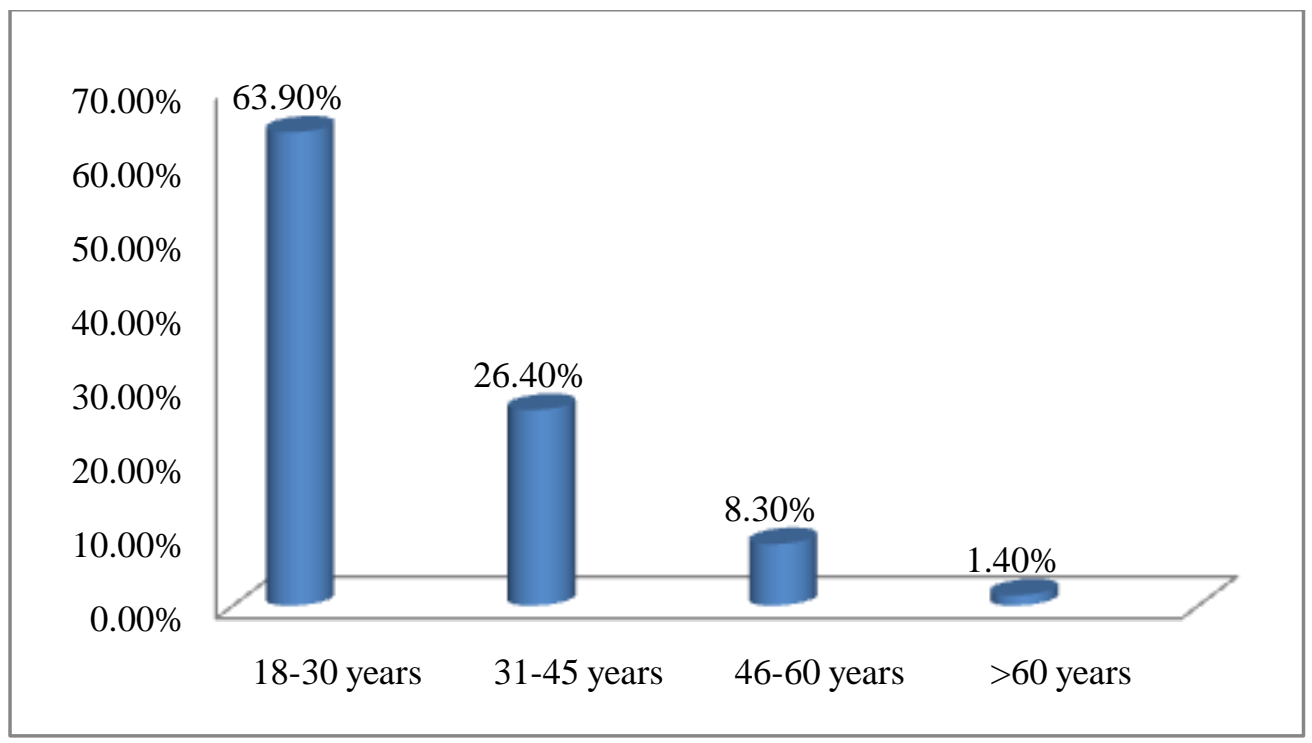

Fig 2:- Age distribution in the study population.

Fig 2 shows the age distribution of the study population. Majority of the patients were in the age group of 18-30 years $(n=46)$ followed by 31-45 years $(n=19)$. Only one patient was more than 60 years of age in our study.

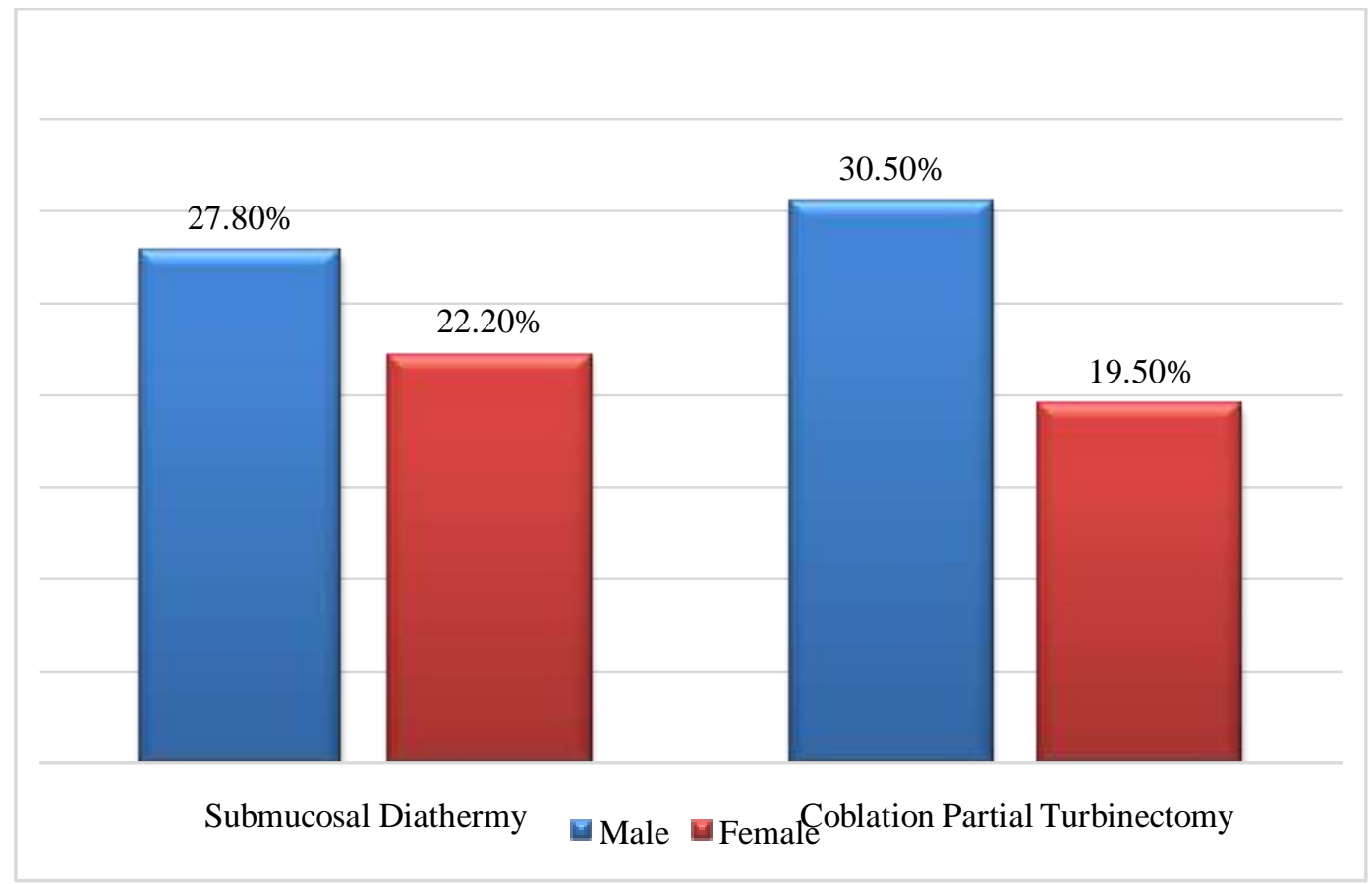

$P$ value -0.8113

Fig 3:- Treatment wise gender distributionin the study population.

Fig. 3 shows the gender distribution in each treatment group. In submucosal diathermy group, $27.80 \%(\mathrm{n}=20)$ were male patients and $22.20 \%(\mathrm{n}=16)$ were female patients. In coblation group, male patients were $30.50 \%(\mathrm{n}=22)$ and female patients were $19.50 \%(\mathrm{n}=14)$. 


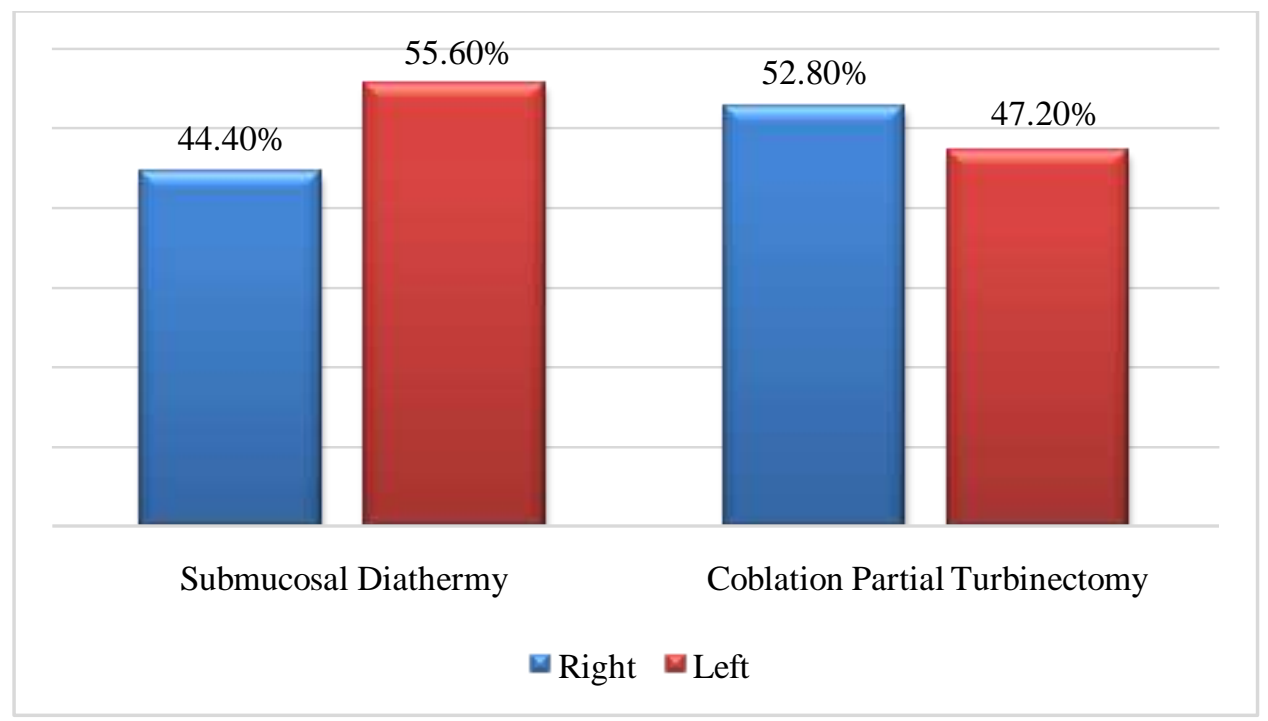

$\mathrm{P}$ value -0.6376

Fig 4:- Side preponderance between the treatment groups in the study population

Fig. 4 shows the side of inferior turbinate hypertrophy in both the treatment groups. In submucosal diathermy group, $44.40 \%(\mathrm{n}=16)$ had right sided inferior turbinate hypertrophy and $55.60 \%(\mathrm{n}=20)$ had left sided inferior turbinate hypertrophy. In coblation group, right sided inferior turbinate hypertrophy was present in $52.80 \%(\mathrm{n}=19)$ patients and left sided inferior turbinate hypertrophy was present in $47.20 \%(n=17)$ patients.

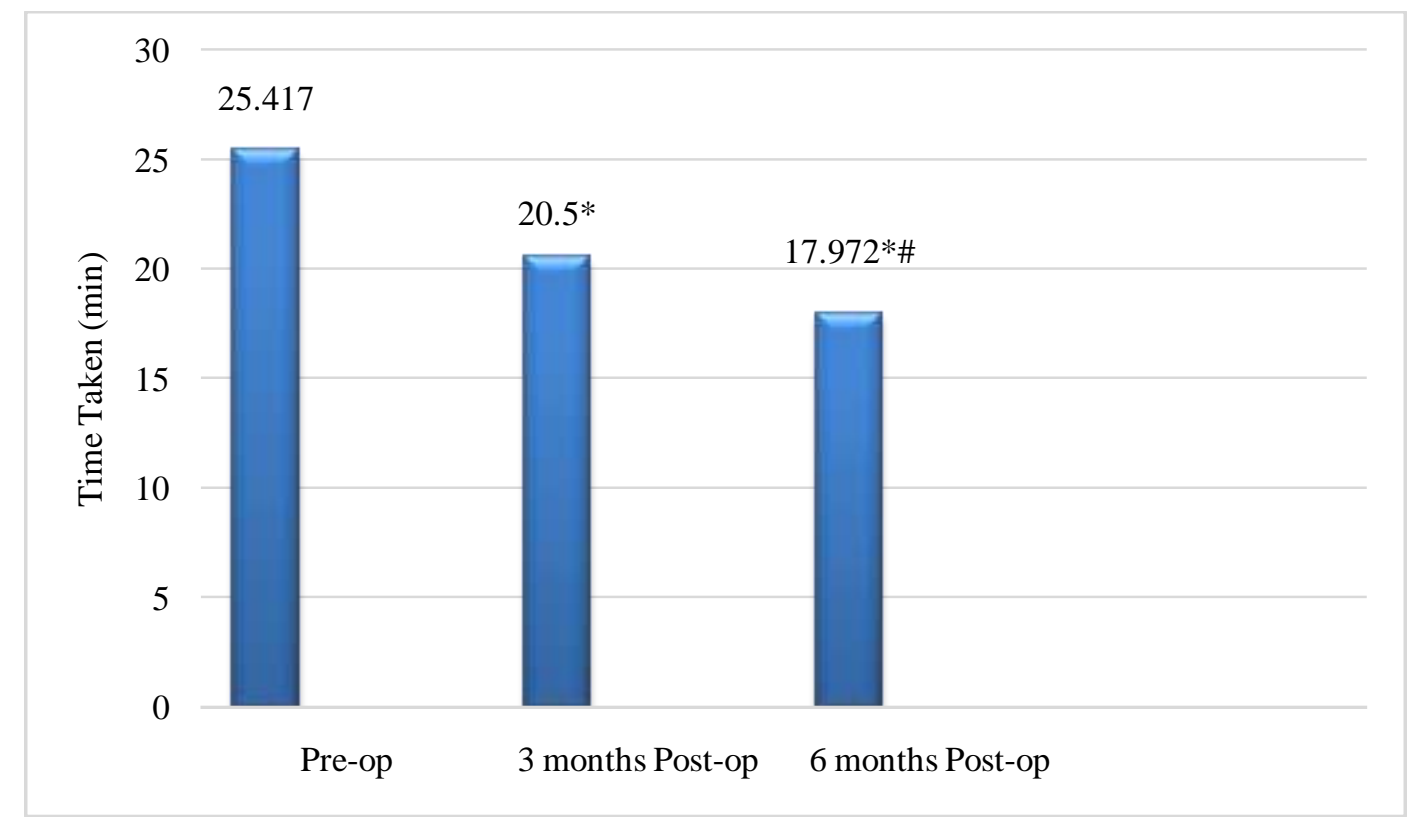

Fig 5:- Effect of submucosal diathermy on nasal mucociliary function.

$*_{p}<0.0001$ between baseline and 3 months and between baseline and 6 months \# $\mathrm{p}<0.0001$ between 3 months and 6 months

Fig. 5 shows the effect of submucosal diathermy on nasal mucociliary function pre-operatively, 3 months and 6 months post-operatively. The time taken pre-operatively was $25.417 \pm 6.429$ (mean $\pm \mathrm{SD}$ ). The time taken 3 months and 6 months post-operatively were $20.5 \pm 4.326$ and $17.972 \pm 3.368$ respectively. It was found that there was a statistically significant improvement in the nasal mucociliary function from pre-op to 3 months and 6 months post- 
op. There was also a significant improvement in nasal mucociliary function when compared between 3 months and 6 months.

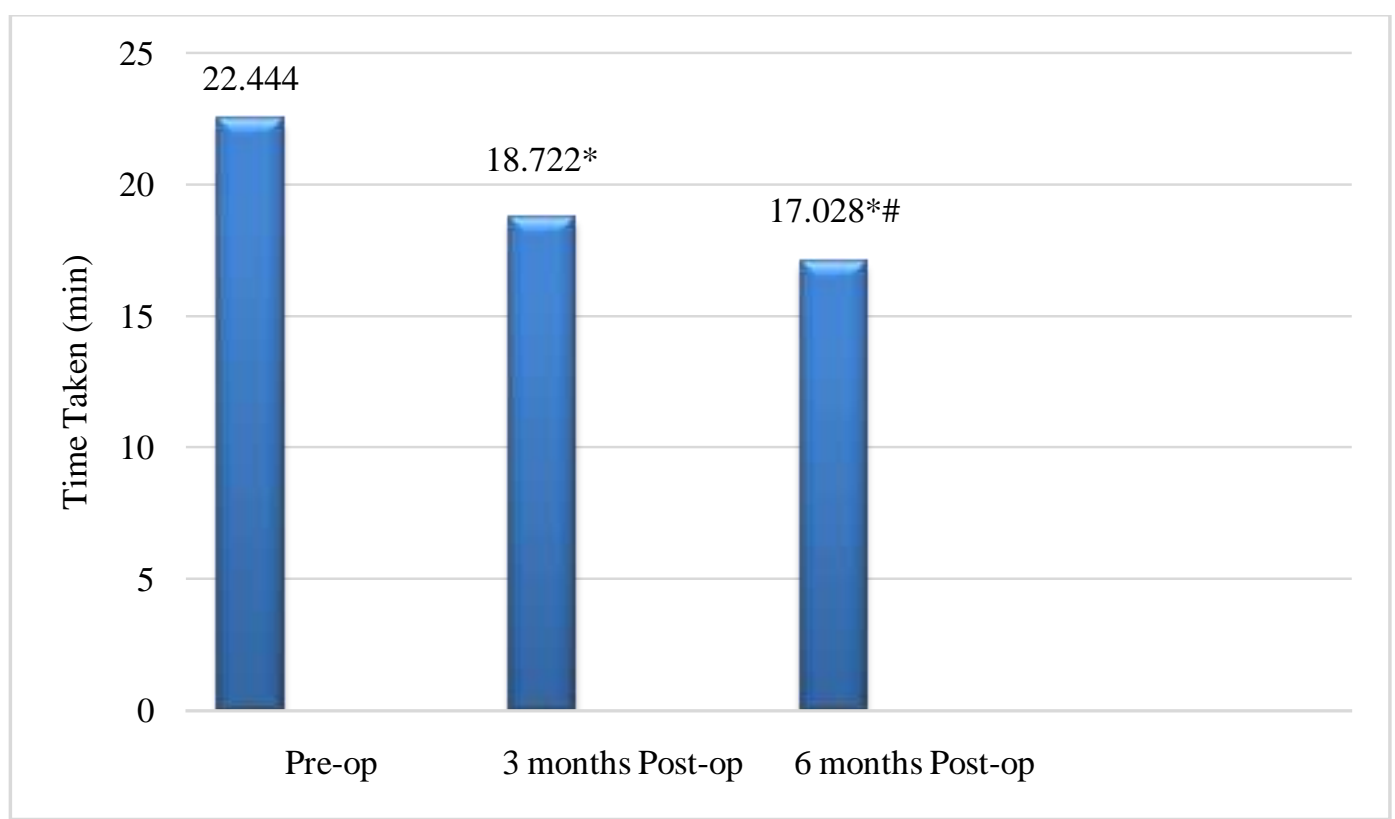

Fig 6:- Effect of coblation partial turbinectomy on nasal mucociliary function.

* $p<0.0001$ between baseline and 3 months and between baseline and 6 months \# $\mathrm{p}<0.0001$ between 3 months and 6 months

Fig. 6 shows the effect of coblation partial turbinectomy on nasal mucociliary function pre-operatively, 3 months and 6 months post-operatively. The time taken pre-operatively was $22.444 \pm 5.326$ (mean $\pm \mathrm{SD}$ ). The time taken 3 months and 6 months post-operatively were $18.722 \pm 4.075$ and $17.028 \pm 3.139$ respectively. It was found that there was a statistically significant improvement in the nasal mucociliary function from baseline to 3 months and 6 months. There was also a statistically significant improvement in nasal mucociliary function when compared between 3 months and 6 months.

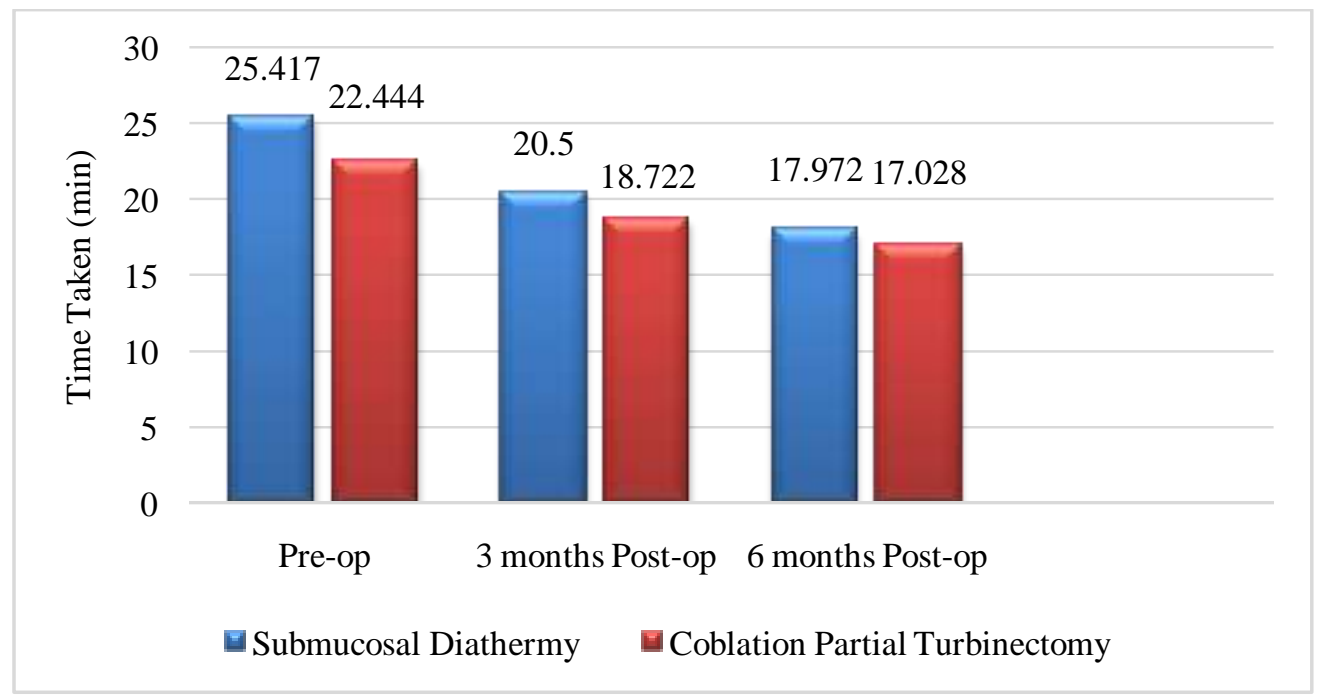

Fig 7:- Comparison of nasal mucociliary function between the treatment groups $\mathrm{p}>0.05$.

Fig. 7 shows the comparative effect of submucosal diathermy vs. coblation partial turbinectomy on nasal mucociliary function at baseline, 3 months and 6 months post-operatively. It was found that there was a no 
statistically significant difference in the improvement in the nasal mucociliary function from baseline to 3 months and 6 months between the two treatment groups.

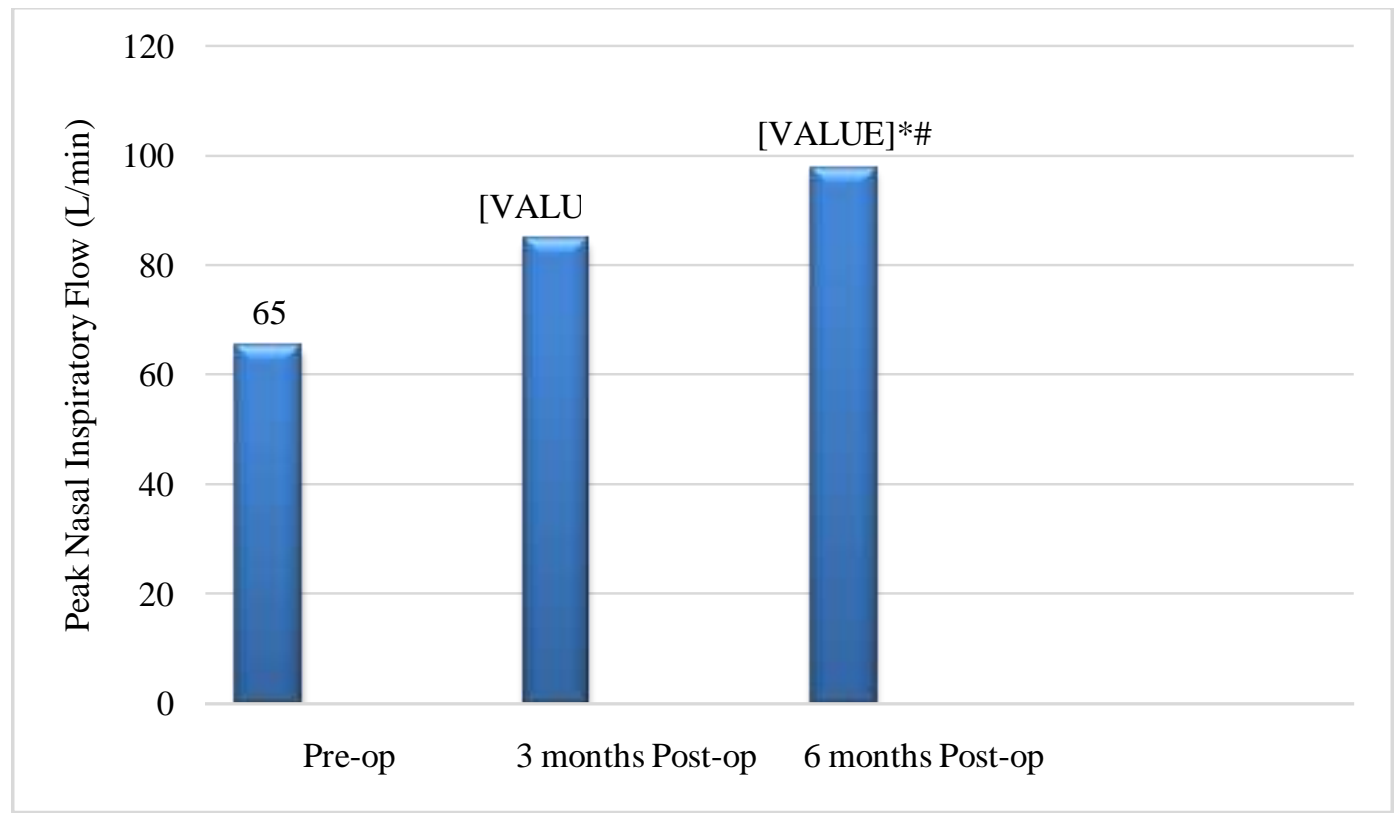

Fig 8:- Effect of submucosal diathermy on Airway patency.

$* p<0.0001$ between baseline and 3 months and between baseline and 6 months \# $\mathrm{p}<0.0001$ between 3 months and 6 months

Fig. 8 shows the effect of submucosal diathermy on PNIF pre-operatively, 3 months and 6 months post-operatively. The flow rate pre-operatively was $65 \pm 11.888$ (mean $\pm \mathrm{SD}$ ). The time taken 3 months and 6 months post-operatively were $84.5 \pm 11.137$ and $97.722 \pm 11.563$ respectively. It was found that there was a statistically significant improvement in PNIF from baseline to 3 months and 6 months. There was also a statistically significant improvement in PNIF when compared between 3 months and 6 months.

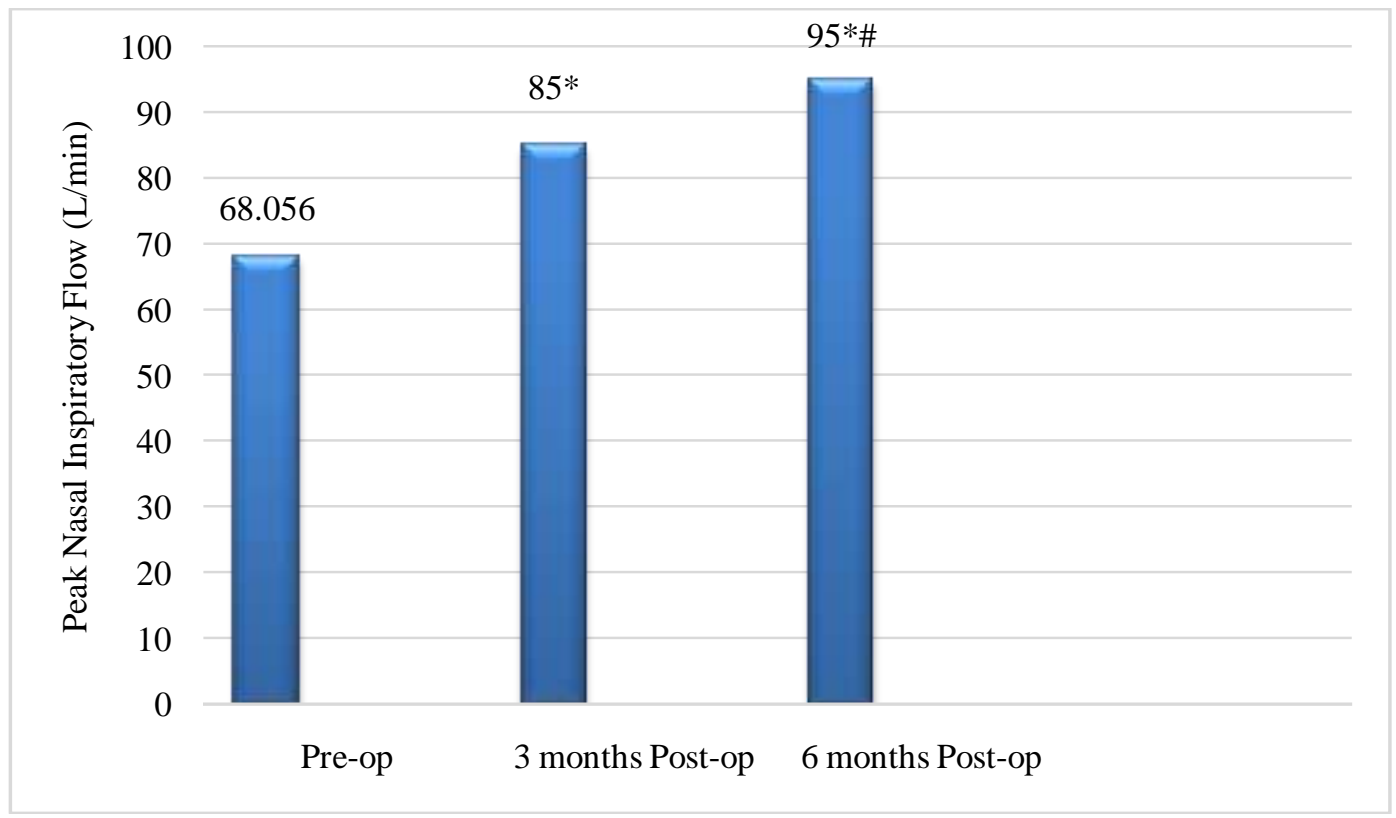

Fig 9:- Effect of Coblation on Airway patency. 
$* \mathrm{p}<0.0001$ between baseline and 3 months and between baseline and 6 months \# $\mathrm{p}<0.0001$ between 3 months and 6 months

Fig. 9 shows the effect of coblation partial turbinectomy on PNIF pre-operatively, 3 months and 6 months postoperatively. The flow rate pre-operatively was $68 \pm 10.907$ (mean \pm SD). The time taken 3 months and 6 months postoperatively were $85 \pm 10.556$ and $95 \pm 11.339$ respectively. It was found that there was a statistically significant improvement in PNIF from baseline to 3 months and 6 months. There was also a statistically significant improvement in PNIF when compared between 3 months and 6 months.

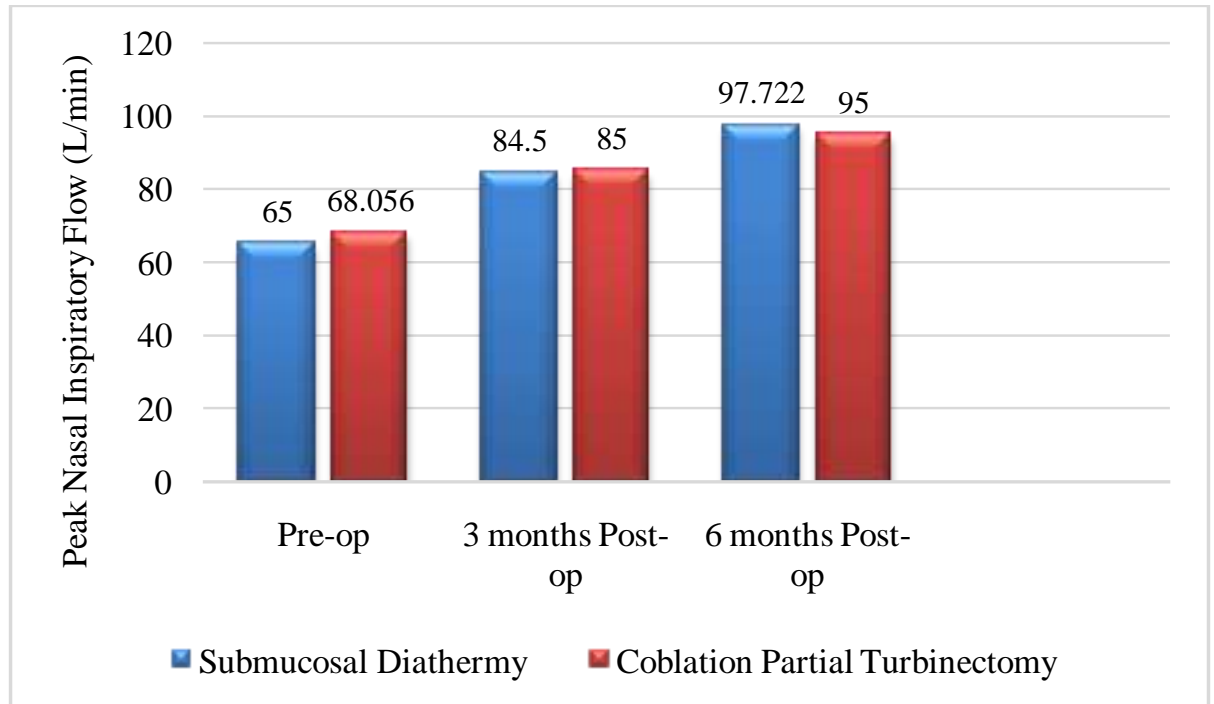

Fig 10:- Comparison of Airway patency between the treatment groups. $\mathrm{p}>0.05$

Fig. 10 shows the comparative effect of submucosal diathermy vs. coblation partial turbinectomy on PNIF preoperatively, 3 months and 6 months post-operatively. It was found that there was a no statistically significant difference in the improvement in the nasal mucociliary function from baseline to 3 months and 6 months between the two treatment groups.

\section{Discussion:-}

Nasal obstruction due to inferior turbinate hypertrophy is a common pathology which can be treated surgically with many available surgical procedures.

In our study we compared the effect of two surgical procedures viz. submucosal diathermy and coblation partial turbinectomy on the nasal mucociliary function using saccharin test and airway patency using PNIF.

As mentioned earlier, the gender distribution in our study was as follows: $58 \%$ were male patients and $42 \%$ were female patients. This is comparable to a study done by Warwick et al39 where the male patients were $57 \%$. In a similar study done by Von Haackeet $\mathrm{al}^{8}, 52 \%$ were male patients which are also similar to our study findings. Another study done by Gindroset al $^{9} 53.3 \%$ were male patients and $46.7 \%$ were female patients which is comparable to our study patients.

The mean age of the study patients is 29.84 years \pm 9.44 years. In the study conducted by Warwick et al ${ }^{10}$ and Von Haacke et $\mathrm{al}^{8}$ the average age was 30.36 years and 30.6 years respectively, which is comparable to our study population. In a study done by Gindroset $\mathrm{al}^{9}$ the mean age of the patients were 32.2 years.

The gender distribution between the two treatment groups was not significant ( $\mathrm{p}$ value -0.8113 ). The distributions of male and female patients in both the groups were the same.

In our study we observed that there was no significant difference ( $p$ value -0.6376 ) in the side of inferior turbinate hypertrophy in both the treatment groups. 
In our study we found that there was an extremely significant $(\mathrm{p}<0.0001)$ difference in the improvement of nasal mucociliary function from baseline when compared with 3 months and 6 months post operatively in both submucosal diathermy and coblation partial turbinectomy groups. We also observed that the improvement in nasal mucociliary function was extremely significant $(\mathrm{p}<0.0001)$ when compared between 3 months and 6 months postsurgery in both the treatment groups.

When we compared the improvement in nasal mucociliary function from baseline to 3 months and 6 months postsurgery, between the two treatment groups, it was observed that there was no significant difference ( $p>0.05$ ) between the two groups suggesting that both the surgical procedures resulted in the same level of improvement in nasal mucociliary function.

In a study conducted by Garzaroet $\mathrm{al}^{11}$, there was a statistically significant $(\mathrm{p}<0.05)$ improvement in the nasal mucociliary function 6 months post-surgery in both the treatment groups. He also compared the improvement of nasal mucociliary function between the two treatment groups and similar to our study it was not significant.

In our study we found that there was an extremely significant $(\mathrm{p}<0.0001)$ difference in the improvement of PNIF from baseline when compared with 3 months and 6 months post operatively in both submucosal diathermy and coblation partial turbinectomy groups. We also observed that the improvement in PNIF was extremely significant $(\mathrm{p}<0.0001)$ when compared between 3 months and 6 months post-surgery in both the treatment groups.

In a study on sixteen patients conducted by Wengrafet $\mathrm{al}^{12}$, there was no improvement in PNIF in the submucosal diathermy group 6 weeks post-surgery which was different from our results. The possible reason for the difference could be due to the difference in the follow-up duration. Another study done by Jones et al ${ }^{13}$ in twenty one patients, they studied the improvement in nasal flow by rhinomanometry and it was seen that there was a statistically significant increase in nasal flow two months post submucosal diathermy. In a similar study conducted by Gindroset $\mathrm{al}^{9}, 30$ patients underwent submucosal cauterization and the patients were followed up at 3 months and 6 months. It was seen that the there was a significant improvement in the nasal flow during follow-up when compared to the preoperative values.

In a study conducted by Farmer et $\mathrm{al}^{14}$, showed statistically significant $(\mathrm{p}=0.0004)$ improvement in the nasal flow 3 months post operatively in patients who underwent coblation turbinectomy.

When we compared the improvement in PNIF from baseline to 3 months and 6 months post-surgery, between the two treatment groups, it was observed that there was no significant difference $(p>0.05)$ between the two groups suggesting that both the surgical procedures resulted in the same level of improvement in PNIF.

In the study done by Fradiset $\mathrm{al}^{15}$, he demonstrated a significant difference $(\mathrm{p}<0.01)$ in the nasal airflow between the two surgical procedures, which is not in line with our study observations. The reason for this non-conformance could be due to (a) the method of measurement of nasal flow. In his study he has used Gertner-Podoshin plate for measuring the nasal air flow, (b) improvement in surgical techniques over the years.

In our study we performed saccharin test to estimate the nasal mucociliary function and PNIF to estimate the airway patency. Use of rhinomanometry would have given more sensitive results.

\section{Conclusion:-}

In the present study we evaluated two widely practised surgical techniques viz. submucosal diathermy and coblation partial turbinectomy for the management of inferior turbinate hypertrophy. Both the surgical techniques showed a statistically significant improvement in nasal mucociliary function and airway patency three months and 6 months post operatively. There was no statistically significant difference between the two surgical procedures. This suggests that both submucosal diathermy and coblation partial turbinectomyare equally effective in improving nasal mucociliary function and airway patency due to inferior turbinate hypertrophy.

\section{Bibliography:-}

1. Smith T, Correa A, Kuo T, Reinisch L. Radiofrequency Tissue Ablation of the Inferior Turbinates Using a Thermocouple Feedback Electrode. The Laryngoscope. 1999;109(11):1760-5. 
2. Li K, Powell N, Riley R, Troell R, Guilleminault C. Radiofrequency Volumetric Tissue Reduction for Treatment of Turbinate Hypertrophy: A Pilot Study. Otolaryngology-Head and Neck Surgery. 1998;119(6):569-73.

3. Nuutinen J. Asymmetry in the Nasal Mucociliary Transport Rate. The Laryngoscope. 1996;106(11):1424-8.

4. Ottaviano G, Scadding G, Scarpa B, Accordi D, Staffieri A, Lund V. Unilateral peak nasal inspiratory flow, normal values in adult population. Rhinology Journal. 2012;50(4):386-92.

5. Utley D, Goode R, Hakim I. Radiofrequency energy tissue ablation for the treatment of nasal obstruction secondary to turbinate hypertrophy. The Laryngoscope. 1999;109(5):683-6.

6. Jackson L, Koch J. Controversies in the Management of Inferior Turbinate Hypertrophy: A Comprehensive Review. Plastic and Reconstructive Surgery. 1999;103(1):300-12.

7. Sapci T, Sahin B, Karavus A, Akbulut U. Comparison of the Effects of Radiofrequency Tissue Ablation, CO2 Laser Ablation, and Partial Turbinectomy Applications on Nasal Mucociliary Functions. The Laryngoscope. 2003;113(3):514-9.

8. vonHaacke N, Hardcastle P. Submucosal Diathermy of the Inferior Turbinate and the Congested Nose. ORL. 1985;47(4):189-93.

9. Gindros G, Kantas I, Balatsouras D, Kaidoglou A, Kandiloros D. Comparison of ultrasound turbinate reduction, radiofrequency tissue ablation and submucosal cauterization in inferior turbinate hypertrophy. European Archives of Oto-Rhino-Laryngology. 2010;267(11):1727-33.

10. Warwick-Brown N, Marks N. Turbinate Surgery: How Effective Is It?. ORL. 1987;49(6):314-20.

11. Garzaro M, Landolfo V, Pezzoli M, Defilippi S, Campisi P, Giordano C et al. Radiofrequency Volume Turbinate Reduction versus Partial Turbinectomy: Clinical and Histological Features. American Journal of Rhinology \& Allergy. 2012;26(4):321-5.

12. Wengraf C, Gleeson M, Siodlak M. The stuffy nose: a comparative study of two common methods of treatment. Clinical Otolaryngology \& Allied Sciences. 2009;11(2):61-8.

13. Jones A, Lancer J. Does submucosal diathermy to the inferior turbinates reduce nasal resistance to airflow in the long term? The Journal of Laryngology \& Otology. 1987;101(5):448-51.

14. Farmer S, Quine S, Eccles R. Efficacy of inferior turbinate coblation for treatment of nasal obstruction. The Journal of Laryngology \& Otology. 2008;123(3):309-14.

15. Fradis M, Danino J, Gaitini L, Gershinski M, Malatskey S, Golz A et al. Inferior Turbinectomy versus Submucosal Diathermy for Inferior Turbinate Hypertrophy. Annals of Otology, Rhinology \& Laryngology. 2000;109(11):1040-5. 